\title{
NATURE OF THE SCALE-INVARIANCE BREAKING TERM \\ IN INELASTIC e-p SCATTERING \\ FROM DGS REPRESENTATION
}

\author{
D. P. MAJUMDAR \\ Harrison M. Randall Laboratory of Physics, \\ The University of Michigan, Ann Arbor, Michigan 48104, USA \\ Received 12 October 1971 \\ Revised manuscript received 4 February 1972
}

\begin{abstract}
Using the DGS representation and the scaling phenomena we suggeat that the nature of the scale breaking term of $\nu W_{2}$ in inelastic e-p scattering, shoul be of the form $G_{2}(\omega) M^{2} / Q^{2}$. Using Regge theory it is then predicted that $G_{2}(\omega)$ would be a constant for large $\omega$, and is estimated to be approximately -0.06 . These predictions, in particular the constancy of the slopes for $\nu W_{2}$ versus $1 / Q^{2}$ plots for large $\omega(\omega>8)$, compare very well with the recent experimental data. Similar results for $W_{1}(\nu, Q 2)$ are also discussed.
\end{abstract}

The SLAC-MIT data [1] on inelastic e-p scattering has revealed some scale breaking effects for the structure function $\nu W_{2}\left(\nu, Q^{2}\right)$. It is found that $\nu W_{2}$ increases with the increase of $Q^{2}$ for a fixed large $\omega(\omega>8)$. The nature of the $Q^{2}$ - dependence of the structure functions (as revealed in fig. 17 of ref. [1]), indicates a very similar characteristic for all large $\omega$, suggesting thereby an underlying simple structure for the scale breaking term. In this paper we demonstrate how this can be understood simply from a Deser-Gilbert Sudarshan [2] (DGS) representation of the forward virtual Compton scattering amplitude, supplemented with scaling and Regge behaviour.

The electroproduction structure functions [3] $W_{2}\left(\nu, Q^{2}\right)$ and $W_{1}\left(\nu, Q^{2}\right)$ are given by the absorptive part of the forward virtual Compton scattering from an unpolarized target. Therefore, since the DGS representation * decribes a causal matrix element of two currents between states having the same four-momentum, it is very suitable [4-6] for studying these inelastic structure functions. We begin by writing a DGS representation for the structure function $W_{2}\left(\nu, Q^{2}\right)$ in the following form

* The DGS representation can be obtained from the following form of the Jost-Lehmann (LJ) representation

$$
F\left(q_{0}, q\right)=\int_{u^{2}<p^{2}} \mathrm{~d} \cdot 3 u \int_{0}^{\infty} \mathrm{d} t^{2} \Psi(t, u) \Delta(\boldsymbol{q}-\mathbf{u}, t) .
$$

Although this representation is a specialization of the JL representation, it has been shown by Nakanishi to hold in every order of perturbation 272

$$
\begin{aligned}
& W_{2}\left(\nu, Q^{2}\right)=Q^{2} \int_{0}^{\infty} \mathrm{d} \lambda \int_{-1}^{1} \mathrm{~d} \beta \sigma_{2}(\lambda, \beta) \\
& \times \delta\left(2 M \beta \nu+M^{2} \beta^{2}-Q^{2}-\lambda\right) .
\end{aligned}
$$

Here we have omitted an $\epsilon\left(M \nu+\beta M^{2}\right)$ factor since we are interested in results for large $\nu$ $(\nu>M)$, and we have included the $Q^{2}$ factor to ensure the kinematic constraint that as $Q^{2}$ goes to zero

$$
\nu W_{2} \underset{Q^{2 \rightarrow 0}}{\longrightarrow}\left(4 \pi^{2} \alpha\right)^{-1} Q^{2} \sigma_{\gamma}(\nu),
$$

where $\sigma_{\gamma}$ is the total photo-absorption crosssection for proton.

It is reasonable with the DGS formalism to assume that the spectral function $\sigma_{2}(\lambda, \beta)$ falls off rapidly as $\lambda$ increases. Then, noting that the expression inside the $\delta$-function can be factorized and remembering that the limits of the $\beta$-integration lies between 1 and -1 , we obtain ** for large $\nu$

** The $\delta$-function can be written as

$$
\begin{aligned}
& \delta\left(2 \beta M \nu+M^{2} \beta^{2}-Q^{2}-\lambda\right)= \\
& \frac{1}{2}\left(\nu^{2}+Q^{2}+\lambda\right)^{-\frac{1}{2}}\left[\delta\left(M \beta+\nu-\sqrt{\nu^{2}+Q^{2}+\lambda}\right)\right. \\
& \quad+\delta\left(M \beta+\nu+\sqrt{\left.\nu^{2}+Q^{2}+\lambda\right)}\right] .
\end{aligned}
$$

For large $\nu$ and $Q^{2}$ and fixed $\omega$ we can write $\sqrt{\nu^{2}+Q^{2}+\lambda}=\sqrt{\nu^{2}+Q^{2}}\left[1+O\left(\lambda / Q^{4}\right)\right]$.

Then since large $\lambda$ values are assumed to be damped out by the spectral function, for first order correction to scaling, $\lambda$ inside the square root can be neglected. 


$$
\begin{aligned}
& W_{2}\left(\nu, Q^{2}\right) \approx \\
& \approx \frac{Q^{2}}{2 M \sqrt{\left(\nu^{2}+Q^{2}\right)}} \int_{0}^{\infty} \mathrm{d} \lambda \sigma_{2}\left(\lambda, \frac{Q^{2}+\lambda}{2 M \nu}-\frac{Q^{4}}{8 M \nu}\right) .
\end{aligned}
$$

We see immediately that in order $\nu W_{2}$ to be finite in the Bjorken limit $\left(\nu, Q^{2} \rightarrow \infty\right.$ with $\omega=$ $2 M \nu / Q^{2}$ fixed) we must have [4]

$\infty$

$\int_{0}^{\infty} \mathrm{d} \lambda \sigma_{2}(\lambda, 1 / \omega)=0$,

for all $\omega$. We have therefore to go to higher order terms in $f(\omega) / \nu$. Thus

$$
\begin{aligned}
& \sqrt{1+Q^{2} / \nu^{2}} \nu W_{2} \underset{\mathrm{B}_{\mathrm{j}}}{\rightarrow} \frac{1}{\omega} \sum_{p=1}^{\infty} \frac{1}{p !(2 M)^{p}} \frac{1}{\nu^{p}-1} \\
& \times \int_{0}^{\infty} \mathrm{d} \lambda\left(\lambda-\frac{M^{2}}{\omega^{2}}\right)^{p} \sigma_{2} p(\lambda, 1 / \omega)
\end{aligned}
$$

where

$\sigma_{2}^{p}(\lambda, 1 / \omega)=\frac{\partial^{p} \sigma_{2}(\lambda, \beta)}{\partial \beta^{p}}$ at $\beta=1 / \omega$.

Since we are interested in the next-to-scale breaking term, we keep only the first two terms in eq. (5) neglecting terms of order $1 / Q^{4}$ and obtain

$$
\sqrt{1+Q^{2} / \nu^{2}} \nu W_{2} \underset{B j}{\longrightarrow} F_{2}(\omega)+\widetilde{G}_{2}(\omega) M^{2} / Q^{2},
$$

where $* * *$,

$F_{2}(\omega)=\frac{1}{2 M \omega} \int_{0}^{\infty} \mathrm{d} \lambda \lambda \sigma_{2}^{\prime}(\lambda, 1 / \omega)$

and

$$
\begin{gathered}
\widetilde{G}_{2}(\omega)=\frac{1}{4 M^{3} \omega^{2}} \int_{0}^{\infty} \mathrm{d} \lambda \lambda^{2} \sigma_{2}^{\prime \prime}(\lambda, 1 / \omega)+ \\
-\frac{1}{2 M \omega^{4}} \int_{0}^{\infty} \mathrm{d} \lambda \lambda \sigma_{2}^{\prime \prime}(\lambda, 1 / \omega) .
\end{gathered}
$$

It is expected that the scale breaking term must be such that it vanishes in the Bjorken limit; but it was not clear a priori what the functional form of this term would be. Eq. (6) gives us a simple $1 / Q^{2}$ form for the breaking term.

\footnotetext{
*** Here we have omitted terms of the type

$\int \mathrm{d} \lambda \sigma_{2}^{\mathrm{P}}(\lambda, 1 / \omega)$, which barring pathological cases would vanish by eq. (4).
}

For an analysis of the data we now expand $\sqrt{1+Q^{2} / \nu^{2}}$ and keep only terms up to $1 / Q^{2}$ and obtain $\dagger[7]$

$\nu W_{2}\left(\nu, Q^{2}\right) \approx F_{2}(\omega)+G_{2}(\omega) M^{2} / Q^{2}$

where

$G_{2}(\omega)=\widetilde{G}_{2}(\omega)-\left(2 / \omega^{2}\right) F_{2}(\omega)$.

We have plotted in fig. 1 the available experimental data [1] for $\nu W_{2}\left(\nu, Q^{2}\right)$ as a function of $1 / Q^{2}$ for several fixed values of $\omega$. Since $\nu W_{2}$ is quite sensitive to the value of $R$, which is not known with good accuracy, we have used only the $6^{\circ}$ data where the errors due to uncertain $R$ will be minimum. We have taken the SLAC-MIT data analyzed for $R=0.18$. The figures show definite indications that the data may fall in straight lines for $Q^{2}>0.5(\mathrm{GeV} / c)^{2}$ as predicted by eq. (9). Note that $\nu W_{2}$ increases $f \dagger[8]$ for a fixed $\omega$ and that the data for different $\omega$ seem to have almost the same slope. We shall presently show why we expect this form a theoretical point of view.

We shall first argue that $G_{2}(\omega)$ has a weak dependence on $\omega$ for at least large values of $\omega$. Our reasoning will essentially be that if $\nu W_{2}$ goes to a constant for large $\omega, \sigma_{2}^{\prime}(\lambda, \beta)$ should have a pole in $\beta$ as $\beta \rightarrow 0$, and then $\sigma_{2}^{\prime \prime}(\lambda, \beta)$ will have a double pole as $\beta \rightarrow 0$. This behaviour will make $F_{2}(\omega)$ and $\widetilde{G}_{2}(\omega)$ independent of $\omega$ for large $\omega$. Finally, for large $\omega, 2 F_{2}(\omega) / \omega^{2} Q^{2}$ can be neglected compared to $F_{2}(\omega)$ leading to the result that $G_{2}(\omega) \approx \widetilde{G}_{2}(\omega)$ (i. e. the slope of $\nu W_{2}$ against $1 / Q^{2}$ plots) be constant and hence same for all large $\omega$.

To obtain more information on $G_{2}(\omega)$, let us now study the Regge limit $\left(\nu \rightarrow \infty, Q^{2}\right.$ fixed) of the structure function. Since $\sigma_{2}(\lambda, \beta)$ falls off for large $\lambda$, and eq. (4) must be satisfied we obtain

T That the breaking of scaling phenomena be of this nature was also predicted from a study of the transverse momentum distributions in the parton model. In that formalism [7] each parton is given a realistic momentum distribution and hence has a component of momentum which is orthogonal in a four-vector sense to the nucleon momentum. This generated a scale-breaking term as a power series in $Q^{2} / \nu^{2}=4 M^{2} / Q^{2} \omega^{2}$, and thus vanishing in the Bjorken limit.

†† This was first pointed out in ref. [7] and also in a phenomenological analysis of the data by Gardiner and Majumdar in ref. [8]. 


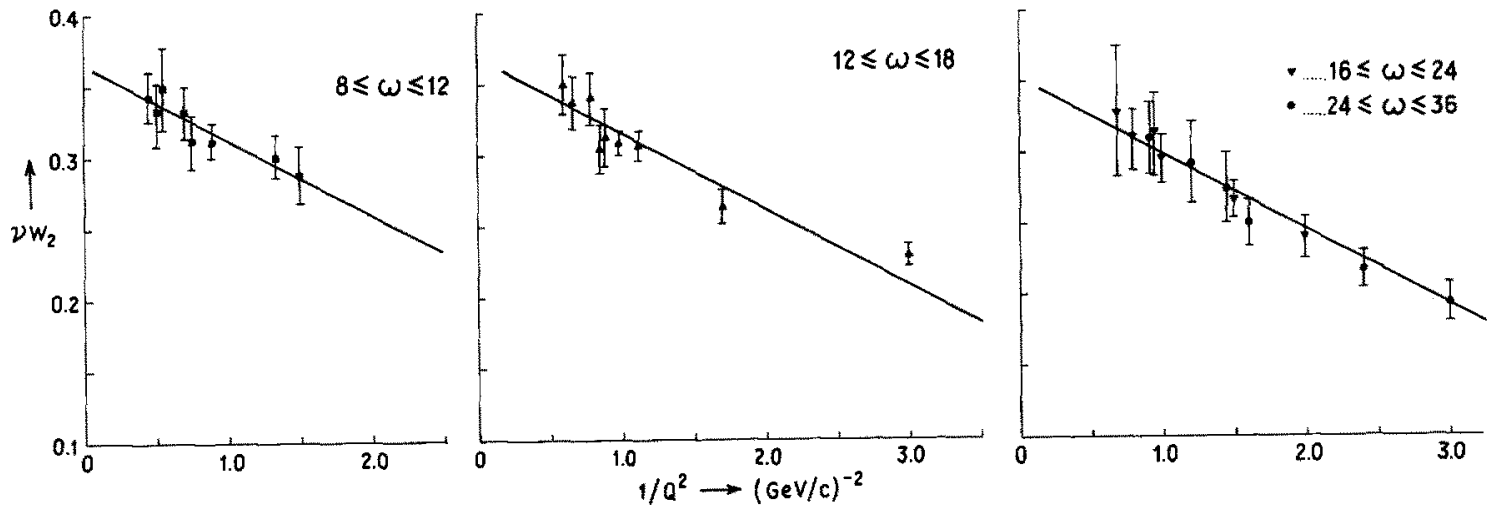

Fig. 1. Values of $\nu W_{2}$ are plotted against $1 / Q^{2}$ for several fixed ranges of $\omega$. The points are taken from ref. [1] for $\theta=6^{\circ}$ measurements analyzed for $R=0.18$. The straight line drawn in all the three figures has the same slope -0.053 .

$$
\begin{aligned}
& W_{2}\left(\nu, Q^{2}\right) \underset{\mathrm{R}}{\underset{p}{\longrightarrow}} \sum_{p=1}^{\infty} \frac{Q^{2}}{(2 M \nu)^{p+1}} \frac{1}{p !} \\
& \times \int_{0}^{\infty} \mathrm{d} \lambda \lambda^{p} \sigma_{2}^{p}\left(\lambda, \frac{Q^{2}}{2 M \nu}\right) .
\end{aligned}
$$

Now, from Regge analysis of the $t$-channel process $\gamma+\gamma \rightarrow \mathrm{N}+\overline{\mathrm{N}}$, one finds [9] that

$W_{2}\left(\nu, Q^{2}\right) \underset{\mathrm{R}}{\longrightarrow} R_{2}\left(Q^{2}\right) \nu^{\alpha-2}$

and

$W_{1}\left(\nu, Q^{2}\right) \underset{\mathrm{R}}{\longrightarrow} R_{1}\left(Q^{2}\right) \nu^{\alpha}$

where $\alpha$ is the leading Regge-trajectory at $t=0$ with natural parity and even charge conjugation. The pomeron with $\alpha=1$ would be the leading trajectory for this process and $\mathrm{P}^{\prime}$ and $\mathrm{A}_{2}$ trajectories both with $\alpha \approx 1 / 2$ can also contribute.

To achieve the result for pomeron dominance, as written down in eq. (12), from our spectral representation eq. (11), we are now led to assume that $\sigma_{2}(\lambda, \beta)$ has a simple pole at $\beta=0$. This behaviour has the additional advantage of producing a constant asymptotic behaviour for $F_{2}(\omega)$ for large $\omega$. Thus we take

$\sigma \neq(\lambda, \beta)=\eta(\lambda) \ln \beta+\tau(\lambda) \beta^{1-\alpha+\chi(\lambda, \beta)}$,

where $\alpha<1$ and $\chi^{\prime}(\lambda, \beta)$ is the least singular part of $\sigma_{2}^{\prime}(\lambda, \beta)$ as $\beta \rightarrow 0$. Eq. (4) then implies that

$\int_{0}^{\infty} \eta(\lambda) \mathrm{d} \lambda=0$,
$\int_{0}^{\infty} T(\lambda) \mathrm{d} \lambda=0$, and

$\int_{0}^{\infty} x(\lambda, \delta)=0$,

when $\delta$ is independent of $\lambda$.

After obtaining the Regge limit, if we let $Q^{2} \rightarrow 0$, we obtain from eq. (2)

$\sigma_{\gamma}(\nu \rightarrow \infty)=\frac{4 \pi^{2} \alpha}{2 M} \int_{0}^{\infty} \mathrm{d} \lambda \eta(\lambda) \ln \lambda$.

After establishing the behaviour of $\sigma_{2}(\lambda, \beta)$ as $\beta \rightarrow 0$, we now go back to the Bjorken limit. The leading contributions are easily obtained from eqs. (7) and (8)

$$
\begin{aligned}
& F_{2}(\omega)=\frac{1}{2 M} \int_{0}^{\infty} \mathrm{d} \lambda \lambda \eta(\lambda)+\frac{1-\alpha}{2 M} \omega_{\omega} \frac{1}{1-\alpha} \int_{0}^{\infty} \mathrm{d} \lambda \lambda \tau(\lambda) \\
& \quad+O(1 / \omega)
\end{aligned}
$$

and

$$
\begin{aligned}
& \widetilde{G}_{2}(\omega)=-\frac{1}{4 M^{3}} \int_{0}^{\infty} \mathrm{d} \lambda \lambda^{2} \eta(\lambda) \\
& -\frac{\alpha(1-\alpha)}{4 M^{3}} \frac{1}{\omega^{1-\alpha}} \int_{0}^{\infty} \mathrm{d} \lambda \lambda^{2} \tau(\lambda)+\mathrm{O}\left(1 / \omega^{2}\right) .
\end{aligned}
$$

Following experimental indications if we now accept that the Bjorken limiting value of $\nu W_{2}$ i. e. $F_{2}(\omega)$ is approximately given by 0.32 (and this seems to be true for $\omega>10$ ), then conside ring only the leading terms of eqs. (19) and (20) we obtain

$$
F_{2}(\omega) \approx \frac{1}{2 M} \int_{0}^{\infty} \mathrm{d} \lambda \lambda \eta(\lambda) \approx 0.32
$$


and

$\widetilde{G}_{2}(\omega) \approx-\frac{1}{4 M^{3}} \int_{0}^{\infty} \mathrm{d} \lambda \lambda^{2} \eta(\lambda)$.

This shows that as $\omega$ increases along with $F_{2}(\omega)$, $\widetilde{G}_{2}(\omega)$ also becomes independent of $\omega$.

To obtain some estimates, we now employ an approximation procedure of saturation at $\lambda=\bar{\lambda}$ of the following kind. Let

$\sigma_{\gamma}(\infty) \approx \frac{4 \pi^{2} \alpha}{2 M} \frac{1}{\bar{\lambda}} \int_{0}^{\infty} \mathrm{d} \lambda \lambda \eta(\lambda)=\frac{4 \pi^{2} \alpha}{\bar{\lambda}} F_{2}(\omega)$.

Since $\sigma_{\gamma}(\infty)$ is approximately [10] $108 \mu \mathrm{b}$, we obtain from eqs. (21) and (22)

$\bar{\lambda}=0.33(\mathrm{GeV})^{2}$

and

$M^{2} \widetilde{G}_{2}(\omega) \approx-\frac{1}{2} \bar{\lambda} F_{2}(\omega)=0.053(\mathrm{GeV} / c)^{2}$.

This is our prediction for the slope ${ }^{t t}$. In figs. $1 \mathrm{a}, 1 \mathrm{~b}$ and $1 \mathrm{c}$ we have drawn a straight-line which has this slope of -0.053 , and the data within errors do seem to fall along this slope. To test the reasonability of our approximation we have calculated $\int \mathrm{d} \lambda \eta(\lambda) \ln \lambda$ with the help of the $\bar{\lambda}$ approximation and also exactly from eq. (18) and found that both give approximately 1.8 for this integral. This means that our approximation of $\ln \lambda$ for eq. (23) is quite good and the rough estimate obtained should not be unreasonable.

Thus froma DGS representation of the virtual forward Compton amplitude and the concepts of scaling and Regge behavior we suggest that the appraoch to scaling in $\nu W_{2}\left(\nu, Q^{2}\right)$ would be of the type $G_{2}(\omega) M^{2} / Q^{2}$ and that the slope of the $\nu W_{2}$ versus $1 / Q^{2}$ graphs be independent of $\omega$ for large $\omega$. We have then argued that the slope be negative and have estimated its value to be $\sim-0.053$. All these predictions are well accommodated by the present experimental data.

Similar analysis can be made for the other

†† If we saturate the integral by a $\delta$-kind of function, say $\eta(\lambda)=-a \delta^{\prime}(\lambda-\bar{\lambda})$, then a reasonable estimate is obtained for $a=0.45$ and $\bar{\lambda}=0.3$ which gives $F_{2}(\omega)=0.24$ and $\widetilde{G}_{2}(\omega)=-0.07$. Again, if we take an exponentially decreasing function with only two parameters say $\eta(\lambda)=\beta(\alpha \lambda-1) \exp (-\alpha \lambda)$ which satisfies eq. (15), then also we obtain reasonable estimates for $\alpha=6$ and $\beta=12.5$ which gives $F_{2}(\omega)=0.2$ and $\tilde{G}_{2}(\omega)=-0.06$. structure function $W_{1}\left(\nu, Q^{2}\right)$. From the kinematical relation between $W_{1}$ and $W_{2}$ we find that in the Bjorken limit

$2 M W_{1} \approx F_{1}(\omega)+\frac{\omega}{1+R} G_{1}(\omega) M^{2} / Q^{2}$,

where

$F_{1}(\omega)=\omega F_{2}(\omega) /(1+R)$

and

$G_{1}(\omega)=\widetilde{G}_{2}(\omega)+\left(2 / \omega^{2}\right) F_{2}(\omega)$.

From eq. (10) we than obtain a prediction between the two slopes of $\nu W_{2}$ and $W_{1}$, namely

$G_{1}(\omega)-G_{2}(\omega)=\left(4 / \omega^{2}\right) F_{2}(\omega)$

and hence, as $\omega$ becomes large, the two slopes, as defined above, will approach each other.

At present it is not possible to test the details of the scale invariance breaking of $W_{1}$ since several data points for a fixed $\omega$ are not available. It has been claimed by Chen [11] that $W_{1}$ exhibits small but definite deviations from scale invariant behaviour. He finds that for $R=$ $0.18,2 M W_{1} /\left[\omega\left(1+Q^{2} / \nu^{2}\right)\right]$ can be accomodated by a scaling curve in the region for $\omega$ from 1.3 to 7.5. This means $G_{1}(\omega)=4 F_{2}(\omega) / \omega 2$ for this kinematical region. Bloom et al.[1] finds no scale breaking of $\nu W_{2}$ for $4 \leqslant \omega \leqslant 6$. That is $G_{2}(\omega)=0$ for this region and hence it agrees well with our prediction eq. (30). For $\omega$ less than 3.5 , it is claimed by Bloom et al. [1] that $\nu W_{2}$ decreases with increase of $Q^{2}$. Eq. (30) then predicts an increase of $G_{1}(\omega)$ for lower $\omega$ values. The verification of this result must await for detailed data at lower $\omega$. Note that for all of our analysis we have assumed $R$ to be constant, and a significant deviation from these predictions would lead us to the question of $Q^{2}$-dependence of $R$.

Several comments are now in order. First it should be stressed that the essential ingredient behind these derivations is the causal representation of the forward virtual Compton amplitude and hence similar conclusions can also be derived from the Jost-Lehmann [12] representation. Then it is natural to expect that the scale breaking term can also be analyzed from considerations of operator-product expansion [13] near the light cone, although the exact nature of the scale breaking term will depend on the dimensions of the singularities. After the completion of our work we came across two preprints [14] which 
made attempts in these directions. These authors find a similar scale breaking term, but in their analysis $G_{2}(\omega)$ derived from the leading light-cone singularity vanishes as $\omega$ goes to infinity, a result in contradiction to the present analysis. Schnitzer [14] has derived an additional scale breaking term from the nonleading light cone singularity of the type $1 /\left(Q^{2}\right)^{b}$, where $b$ is 1 for canonical dimensions only.

In conclusion we may add that our analysis, although dependent on the particular representation chosen here, and perhaps not as rigorous as it should be, are essentially the results of a causal representation, and hence it would be very interesting to see how these results compare with the future experimental data. It goes without saying that a better experimental determination of the scale breaking effects for large $\omega$ would be very crucial from a theoretical point of view. Details of this work including higher order terms and further comparisons with investigations from other theoretical points of view will be published elsewhere.

I would like to thank Professor J. D. Bjorken for suggesting this investigation and Professor Y. Tomozawa for many helpful discussions.

\section{References}

[1] E. D. Bloom et al., Fifteenth Intern. Conf. on High energy physicis, Kiev, USSR (1970). SLACPUB-796.

[2] S. Deser, W. Gilbert and E. C. G. Sudarshan, Phys. Rev. 117 (1960) 266; 115 (1959) 731;

N. Nakanishi, Progr. Theoret. Phys. 26 (1961) 337.

[3] S. Drell and J. Walecka, Ann. Phys. (N. Y.) 28 (1964) 18; J. D. Bjorken, Phys. Rev. 179 (1969) 1547.

[4] R. A. Brandt, Phys, Rev. Letters 22 (1969) 1149.

[5] L.S. Brown, Lectures in Theoretical Physics XII, eds. K. T. Mahanthappa and W. R. Brittin (Gordon and Breach, New York).

[6] J. M. Cornwall, D. Corrigan and R. E. Norton, Phys. Rev. D3 (1971) 536; A. Suri, Phys. Rev. D4 (1971) 570.

[7] C. W. Gardiner and D. P. Majumdar, Phys. Rev. D2 (1970) 2040.

[8] C. W. Gardiner and D. P. Majumdar, Syracuse University preprint SU-1206-236 (1970) unpublished.

[9] H. D. I. Abarbane1, M. L. Goldberger and S. B. Treiman, Phys. Rev. Letters 22 (1969) 500.

[10] E. D. Bloom et al., SLAC-PUB-653 (1969).

[11] Fong-Ching Chen, CERN preprint Th. 1285.

[12] R. Jost and H. Lehmann, Nuovo Cimento 5 (1957) 1598.

[13] K. Wilson, Rapporter's talk at 1971 Intern. Symp. on Electron and photon interactions at high energies, Cornell University.

[14] H. J. Schnitzer, Phys. Rev. to be published; K. T. Mahanthappa and T. Yao, ICTP (Trieste) preprint. 